\title{
ON A RESIDUE OF COMPLEX FUNCTIONS IN THE THREE-DIMENSIONAL EUCLIDEAN COMPLEX VECTOR SPACE
}

\author{
BRANKO SARIĆ
}

Received 2 December 2001

\begin{abstract}
In the introductory part of this paper, a notion of absolute integral sums of a complex function, which is more general with respect to that of an integral and integral sums of ordinary integral calculus, is defined. Throughout the main part of the paper, an attempt has been made to generalize, on the basis of redefining the notion of a complex function residue, some of the fundamental results of Cauchy's calculus of residues of analytic functions. The foundation stone of the whole theory is the total value of an improper integral of complex functions.
\end{abstract}

2000 Mathematics Subject Classification: 30G30, 32A30, 30E20.

\section{Introduction}

1.1. Notations and definitions. In this paper, we mean by a three-dimensional complex vector space $\vec{r}=x \vec{e}_{1}+i y \vec{e}_{2}+\chi \vec{n}$ (i denotes the imaginary unit) a vector space of the definite Euclidean metric $d s^{2}=d \vec{r} \cdot d \vec{r}^{*}=d x^{2}+$ $d y^{2}+d x^{2}$, where $\vec{r}^{*}=x \vec{e}_{1}-i y \vec{e}_{2}+x \vec{n}$ is the complex conjugate of $\vec{r}$ and $\vec{n}$ is a normal vector of the unit length of $\vec{\varrho}=x \vec{e}_{1}+i y \vec{e}_{2}$ ( $\vec{\varrho}$ is a two-dimensional complex vector plane of the definite Euclidean metric $d q^{2}=d \vec{\varrho} \cdot d \vec{\varrho}^{*}=$ $d x^{2}+d y^{2}$ ). The one-to-one map $z^{*}=x-i y$ and $z=x+i y$ maps the twodimensional complex vector plane $\vec{\varrho}=z^{*} \vec{w}_{1}+z \vec{w}_{2}\left(\vec{w}_{l} \cdot \vec{w}_{j}=\delta_{l j}\right.$, where $\delta_{l j}$ is the Kronecker delta, more precisely, the identity $2 \times 2$ matrix) into itself $\left(\underline{\varrho}=x \vec{e}_{1}+i y \vec{e}_{2}\right)$, with the so-called Jacobian of transformation

$$
J=\left|\begin{array}{ll}
\frac{\partial z^{*}}{\partial x} & \frac{\partial z^{*}}{\partial i y} \\
\frac{\partial z}{\partial x} & \frac{\partial z}{\partial i y}
\end{array}\right|=\left|\begin{array}{rr}
1 & -1 \\
1 & 1
\end{array}\right|=2
$$

If $\vec{r}_{A}$ is a position vector of an arbitrary point $A$, then the set $\vec{r}_{G}$ of points $\vec{r}_{A}$ defines, in the three-dimensional Euclidean complex vector space $\vec{r}$, some arbitrary domain $G: \vec{r}_{G}=\left\{\vec{r}_{A}: A \in G\right\}$. If the domain $\vec{r}_{G}$ bounded by a contour surface $\vec{r}_{g}$ in $\vec{r}$ is subdivided by planes, which are parallel to the coordinate planes, into $k_{1}$ elemental subdomains $\Delta_{j_{1}} \vec{r}_{G}$ bounded by the elemental contour surfaces $\Delta_{j_{1}} \vec{r}_{g}\left(j_{1}=2, \ldots, k_{1}\right)$, then every subdomain $\Delta_{j_{1}} \vec{r}_{G}$ of $\vec{r}_{G}$ 
can be further subdivided into new subdomains $\Delta_{j_{1}, j_{2}} \vec{r}_{G}\left(j_{2}=2, \ldots, k_{2}\right)$. (If an infinite process of a subdivision of the domain $\vec{r}_{G}$ in $\vec{r}$ leads to any point $\vec{r}_{N}$ of $\vec{r}_{G}: \lim _{n \rightarrow+\infty} \Delta_{j_{1}, \ldots, j_{n}} \vec{r}_{G}=\vec{r}_{N}$, then the subdivision $D_{n} \vec{r}_{G}$ is said to be an evenly spaced one.) Let $\Delta_{j_{1}, \ldots, j_{n}} \sigma$ and $\Delta_{j_{1}, \ldots, j_{n}} v$ denote numerous measures of an area of $\Delta_{j_{1}, \ldots, j_{n}} \vec{r}_{g}$ and a volume of $\Delta_{j_{1}, \ldots, j_{n}} \vec{r}_{G}$, respectively. Then $d_{\vec{r}_{N}} v=\lim _{n \rightarrow+\infty} \Delta_{j_{1}, \ldots, j_{n}} v=0$ and $d_{\vec{r}_{N}} \vec{\sigma}=\lim _{n \rightarrow+\infty} \vec{n}_{\vec{r}_{\Delta_{1}, \ldots, j_{n}} \vec{r}_{g}} \Delta_{j_{1}, \ldots, j_{n}} \sigma=\overrightarrow{0}$ $\left(\vec{n}_{\vec{r}_{j_{1}, \ldots, j_{n}} \vec{r}_{g}}\right.$ is a normal vector of the unit length of $\Delta_{j_{1}, \ldots, j_{n}} \vec{r}_{g}$ at an arbitrary point $\vec{r}_{\Delta_{1}, \ldots, j_{n} \vec{r}_{g}}$ ) are an infinitesimal volume element and an infinitesimal surface element at a point $\vec{r}_{N}$ of the domain $\vec{r}_{G}$, respectively.

Since vectors $\Delta_{\vec{r}_{j_{1}, \ldots, j_{n}} \overrightarrow{r g}} \vec{\sigma}=\vec{n}_{\vec{r}_{j_{1}, \ldots, j_{n}} \vec{r}_{g}} \Delta_{j_{1}, \ldots, j_{n}} \sigma$, at an arbitrary point $\vec{r}_{\Delta_{j_{1}, \ldots, j_{n}} \vec{r}_{g}}$ of a part of the elemental contour surface $\Delta_{j_{1}, \ldots, j_{n}} \vec{r}_{g}$ separating two elemental subdomains, have opposite orientations, for every evenly spaced subdivision:

$$
D_{n} \vec{r}_{G}=\left\{\triangle_{j_{1}, \ldots, j_{l}} \vec{r}_{G}: j_{l}=2, \ldots, k_{l}(l=1,2, \ldots, n)\right\}
$$

of $\vec{r}_{G}$ bounded by $\vec{r}_{g}$, it follows that

$$
\lim _{n \rightarrow+\infty} \sum_{j_{1}=2}^{k_{1}} \sum_{j_{2}=2}^{k_{2}} \cdots \sum_{j_{n}=2}^{k_{n}} \Delta_{\vec{r}_{\Delta_{1}}, \ldots, j_{n} \overrightarrow{r_{g}}} \vec{\sigma}=\sum_{\vec{r}_{N} \in \vec{r}_{g}} d_{\vec{r}_{N}} \vec{\sigma}
$$

The infinite sum $\sum_{\vec{r}_{N} \in \vec{r}_{g}} d_{\overrightarrow{r_{N}}} \vec{\sigma}$ of zero vectors $\sum_{\vec{r}_{N} \in \vec{r}_{g}} d_{\vec{r}_{N}} \vec{\sigma}=\overrightarrow{0} \times \infty$, as an indefinite expression, in this acute case reduces to the vector $\vec{P}$ whose intensity is equal to the area of $\vec{r}_{g}: \sum_{\vec{r}_{N} \in \vec{r}_{g}} d_{\vec{r}_{N}} \vec{\sigma}=\vec{P}$.

For an arbitrary scalar-valued function $f(\vec{r})$ defined and bounded on the domain $\vec{r}_{G}$ of the three-dimensional Euclidean complex vector space $\vec{r}$, the following holds:

$$
\lim _{n \rightarrow+\infty} \sum_{j_{1}=2}^{k_{1}} \sum_{j_{2}=2}^{k_{2}} \cdots \sum_{j_{n}=2}^{k_{n}} f\left(\vec{r}_{\Delta_{1}, \ldots, j_{n}} \vec{r}_{g}\right) \triangle_{\vec{r}_{\Delta_{1}, \ldots, j_{n}} \overrightarrow{r_{g}}} \vec{\sigma}=\sum_{\vec{r}_{N} \in \vec{r}_{g}} f\left(\vec{r}_{N}\right) d_{\vec{r}_{N}} \vec{\sigma},
$$

where $f\left(\vec{r}_{\Delta_{j_{1}, \ldots, j_{n}} \vec{r}_{g}}\right)$ are values of $f(\vec{r})$ at arbitrary points of parts of the elemental contour surfaces $\Delta_{j_{1}, \ldots, j_{n}} \vec{r}_{g}$ separating two elemental subdomains, as well as at arbitrary points of $\Delta_{j_{1}, \ldots, j_{n}} \vec{r}_{g}$ belonging to $\vec{r}_{g}$. If $f(\vec{r})$ is a Riemannintegrable function over the domain $\vec{r}_{G}$, then for any evenly spaced subdivision $D_{n} \vec{r}_{G}$ of $\vec{r}_{G}$ and any choice of points $\vec{r}_{\Delta_{1}, \ldots, j_{n}} \vec{r}_{g}$, there exists a unique limiting value

$$
\lim _{n \rightarrow+\infty} \sum_{j_{1}=2}^{k_{1}} \sum_{j_{2}=2}^{k_{2}} \cdots \sum_{j_{n}=2}^{k_{n}} f\left(\vec{r}_{\Delta_{j_{1}, \ldots, j_{n}} \vec{r}_{g}}\right) \triangle_{\vec{r}_{\Delta_{1}, \ldots, j_{n}} \overrightarrow{r_{g}}} \vec{\sigma}=\iint_{\vec{r}_{g}}^{\cup} f(\vec{r}) d \vec{\sigma}
$$

The symbol $\iint_{\overrightarrow{r_{g}}}^{\cup}$ denotes an integration over the closed contour surface $\vec{r}_{g}$, in this case in the positive mathematical direction. 
To sum up, for a function $f(\vec{r})$, which is Riemann-integrable over $\vec{r}_{G}$, the infinite sum of zero vectors $\sum_{\vec{r}_{N} \in \vec{r}_{g}} f\left(\vec{r}_{N}\right) d_{\vec{r}_{N}} \vec{\sigma}=\infty \times \overrightarrow{0}$, as an indefinite expression, is just equal to the integral of $f(\vec{r})$

$$
\sum_{\vec{r}_{N} \in \vec{r}_{g}} f\left(\vec{r}_{N}\right) d_{\overrightarrow{r_{N}}} \vec{\sigma}=\iint_{\vec{r}_{g}}^{\cup} f(\vec{r}) d \vec{\sigma} .
$$

Accordingly, we are able to redefine, more exactly, to generalize the notion of Riemann integral sums as follows.

DEFINITION 1.1. Absolute integral sums of a scalar-valued function $f(\vec{r})$, defined on the domain $\vec{r}_{G}$ bounded by a contour surface $\vec{r}_{g}$ in the three-dimensional Euclidean complex vector space $\vec{r}$, are by definition

$$
\sum_{\overrightarrow{r_{N}} \in \vec{r}_{g}} f\left(\vec{r}_{N}\right) d_{\vec{r}_{N}} \vec{\sigma}, \quad \sum_{\vec{r}_{N} \in \vec{r}_{G}} f\left(\vec{r}_{N}\right) d_{\vec{r}_{N}} v
$$

DEFINITION 1.2. Absolute integral sums of a vector-valued function $\vec{F}(\vec{r})$, defined on the domain $\vec{r}_{G}$ bounded by a contour surface $\vec{r}_{g}$ in the three-dimensional Euclidean complex vector space $\vec{r}$, are by definition

$$
\begin{gathered}
\sum_{\vec{r}_{N} \in \vec{r}_{g}} \vec{F}\left(\vec{r}_{N}\right) \cdot d_{\vec{r}_{N}} \vec{\sigma}, \quad \sum_{\vec{r}_{N} \in \vec{r}_{g}} d_{\vec{r}_{N}} \vec{\sigma} \times \vec{F}\left(\vec{r}_{N}\right), \\
\sum_{\vec{r}_{N} \in \vec{r}_{G}} \vec{F}\left(\vec{r}_{N}\right) d_{\vec{r}_{N}} v .
\end{gathered}
$$

1.2. The purpose of the paper. Thus far, little attention has apparently been paid to the general case in which functions of a complex variable have an infinity of singularities. But in various applications, for example, in [1, 3], it was essential to analyze, on the basis of the residual calculus theory, this class of functions, so that the goal of this paper is to establish a general theory of residual calculus whose results would be slightly more general in comparison with the fundamental results of Cauchy's calculus of residues. Since functions, which are regular ones in an extended complex plane except at infinitely but countable many points, do not belong to the functional space of either analytic or nonanalytic functions, it follows that the notion of a residue of the class of the aforementioned functions cannot be defined in the same way as was done in the case of a class of analytic as well as nonanalytic functions. Accordingly, it is necessary to redefine the notion of a complex function residue, more precisely, to generalize it. This notion, as is well known, was first generalized by Poor [4, 5] (taken over from [3]), see, for example, [3, Definitions 1 and 2, pages 38-39]. 
1.3. The outline of the rest of the paper. In Section 2.1, we will define, on the basis of the definitions from the introductory part of the paper, a spatial differentiability of a complex function. Our variant of the spatial derivative of a complex function will turn out to be very convenient in defining a residue of this general class of functions as it was done in Section 2.2. We define a potential of a point with respect to a contour surface of integration. Thereafter, in the same subsection, we define a total value of an improper integral as a sum of Cauchy's principal value and Jordan's singular value. Note, as peculiarity, that the total value of an improper integral is not a unique defined value in the general case [6]. This subsection ends with an example that illustrates a notion of a singular-analytic function.

\section{Main results}

2.1. On spatial differentiability of a complex function. Let $f(\vec{r})$ be an arbitrary uniform scalar-valued function defined on some domain $\vec{r}_{G}$ of the threedimensional Euclidean complex vector space $\vec{r}=\vec{\varrho}+\varkappa \vec{n}$ as an ambient space of the two-dimensional Euclidean complex vector space $\vec{\varrho}$. The following definition is an obvious consequence of the integral equality from the definition of the spatial derivative of $f(\vec{r})$, see, for example, [2, Definition 2, page 291].

DEFINITION 2.1. A scalar-valued function $f(\vec{r})$ is spatially differentiable over the domain $\vec{r}_{G}$ bounded by a contour surface $\vec{r}_{g}$ in the three-dimensional Euclidean complex vector space $\vec{r}$ if and only if, for every evenly spaced subdivision $D_{n} \vec{r}_{G}$ and every elemental subdomain $\Delta_{j_{1}, \ldots, j_{n}} \vec{r}_{G}$ of $\vec{r}_{G}$, the sequence of reduced absolute integral sums:

$$
\vec{A}_{\Delta_{j_{1}, \ldots, j_{n}} \vec{r}_{g}}=\frac{1}{\Delta_{j_{1}, \ldots, j_{n}} v} \sum_{\vec{r}_{N} \in \Delta j_{1}, \ldots, j_{n} \overrightarrow{r_{g}}} f\left(\vec{r}_{N}\right) d_{\overrightarrow{r_{N}}} \vec{\sigma}
$$

converges to

$$
\lim _{\overrightarrow{r_{g}} \rightarrow \vec{r}_{N}} \frac{1}{V} \sum_{\vec{r}_{N} \in \vec{r}_{g}} f\left(\vec{r}_{N}\right) d_{\vec{r}_{N}} \vec{\sigma}=\lim _{n \rightarrow+\infty} \vec{A}_{\Delta_{j_{1}, \ldots, j_{n}} \vec{r}_{g}}=\vec{A}_{\vec{r}_{N}},
$$

where $V=\sum_{\vec{r}_{N} \in \vec{r}_{G}} d_{\vec{r}_{N}} v$.

The domain $\vec{r}_{G}$ in the three-dimensional Euclidean complex vector space $\vec{r}$, such that at all points of $\vec{r}_{G}$, a scalar-valued function $f(\vec{r})$ is spatially differentiable, is a regular domain of $f(\vec{r})$. The points $\vec{r}_{N}$ in $\vec{r}$, at which $f(\vec{r})$ is not differentiable, are singular points of $f(\vec{r})$, and the domain $\vec{r}_{G}$, such that $f(\vec{r})$ is differentiable almost everywhere over $\vec{r}_{G}$, is a singular domain of $f(\vec{r})$. The singular points $\vec{r}_{N}$ of $\vec{r}_{G}$, at which $f(\vec{r})$ is bounded, are apparent singular points of $f(\vec{r})$. The singular domain $\vec{r}_{G}$, such that $f(\vec{r})$ is bounded on $\vec{r}_{G}$, is an apparent singular domain of $f(\vec{r})$. 
If $f(\vec{r})$ is spatially differentiable over $\vec{r}_{G}$, then for an arbitrary evenly spaced subdivision $D_{n} \vec{r}_{G}$ and an arbitrary elemental subdomain $\Delta_{j_{1}, \ldots, j_{n}} \vec{r}_{G}$ of the regular domain $\vec{r}_{G}$, it follows from equality (2.1) of Definition 2.1 that

$$
\sum_{\vec{r}_{N} \in \Delta_{j_{1}, \ldots, j_{n}} \overrightarrow{r_{g}}} f\left(\vec{r}_{N}\right) d_{\overrightarrow{r_{N}}} \vec{\sigma}=\vec{A}_{\Delta_{j_{1}, \ldots, j_{n}} \vec{r}_{g}} \Delta_{j_{1}, \ldots, j_{n}} v
$$

In view of the fact that surface elements $d_{\vec{r}_{N}} \vec{\sigma}$, at every point $\vec{r}_{N}$ of the part of an elemental contour surface $\Delta_{j_{1}, \ldots, j_{n}} \vec{r}_{g}$ separating two elemental subdomains, are oppositely directed, for every level of the evenly spaced subdivision $D_{n} \vec{r}_{G}$ of $\vec{r}_{G}$,

$$
\sum_{j_{1}=2}^{k_{1}} \sum_{j_{2}=2}^{k_{2}} \cdots \sum_{j_{n}=2}^{k_{n}} \sum_{\vec{r}_{N} \in \Delta j_{1}, \ldots, j_{n} \vec{r}_{g}} f\left(\vec{r}_{N}\right) d_{\overrightarrow{r_{N}}} \vec{\sigma}=\sum_{\vec{r}_{N} \in \vec{r}_{g}} f\left(\vec{r}_{N}\right) d_{\vec{r}_{N}} \vec{\sigma}
$$

On the other hand, on the basis of the convergence of reduced absolute integral sums, more precisely, from equality (2.2) of Definition 2.1, we obtain

$$
\lim _{n \rightarrow+\infty} \sum_{j_{1}=2}^{k_{1}} \sum_{j_{2}=2}^{k_{2}} \cdots \sum_{j_{n}=2}^{k_{n}} \vec{A}_{\Delta_{j_{1}}, \ldots, j_{n} \vec{r}_{g}} \Delta_{j_{1}, \ldots, j_{n}} v=\sum_{\vec{r}_{N} \in \vec{r}_{G}} \vec{A}_{\vec{r}_{N}} d_{\vec{r}_{N}} v
$$

Hence, these last three expressions give

$$
\sum_{\overrightarrow{r_{N}} \in \vec{r}_{g}} f\left(\vec{r}_{N}\right) d_{\vec{r}_{N}} \vec{\sigma}=\sum_{\vec{r}_{N} \in \vec{r}_{G}} \vec{A}_{\vec{r}_{N}} d_{\vec{r}_{N}} v
$$

If $f(\vec{r})$ is defined and continuous on the domain $\vec{r}_{G}$ bounded by a contour surface $\vec{r}_{g}$, more precisely, is integrable over $\vec{r}_{G}$, which is its regular domain in the sense of Definition 2.1, then it follows from (2.6) and (1.6) that

$$
\iint_{\vec{r}_{g}}^{\cup} f(\vec{r}) d \vec{\sigma}=\sum_{\vec{r}_{N} \in \vec{r}_{G}} \vec{A}_{\vec{r}_{N}} d_{\vec{r}_{N}} v
$$

that is,

$$
\lim _{\overrightarrow{r_{g} \rightarrow \vec{r}_{N}}} \frac{1}{V} \iint_{\overrightarrow{r_{g}}}^{\cup} f(\vec{r}) d \vec{\sigma}=\vec{A}_{\vec{r}_{N}}
$$

where $V=(1 / 3) \iint_{\vec{r}_{g}}^{\cup} \vec{r} \cdot d \vec{\sigma}$.

If a vector-valued function $\vec{A}(\vec{r})\left(\vec{A}\left(\vec{r}_{N}\right)=\vec{A}_{\vec{r}_{N}}\right)$ is also defined and continuous on $\vec{r}_{G}$, more precisely, is integrable over $\vec{r}_{G}$, then (2.7) reduces to

$$
\iint_{\vec{r}_{g}}^{\cup} f(\vec{r}) d \vec{\sigma}=\iiint_{\vec{r}_{G}} \vec{A}(\vec{r}) d v
$$


Clearly, in this case, $\vec{A}(\vec{r})$ is a vector-valued function of the spatial derivative of a continuous function $f(\vec{r}): \vec{A}(\vec{r})=\nabla \cdot f(\vec{r})$, and $\nabla$ is the so-called Hamiltonian operator of spatial differentiability, see [2].

In view of the fact that $d \vec{\sigma}=d \vec{\varrho} \times d x \vec{n}+d z^{*} d z \vec{n}$ and $d v=d \vec{r} \cdot d \vec{\sigma}=$ $d z^{*} d z d x$ if the function $f(\vec{r})$ is defined and continuous on (integrable over) the bounded domain $\vec{r}_{G}$ in the three-dimensional Euclidean complex vector space $\vec{r}$ and possesses defined and continuous (integrable) partial derivatives on (over) $\vec{r}_{G}$, then it follows from (2.9) that

$$
\begin{aligned}
\iint_{\vec{r}_{g}}^{\cup} f(\vec{r}) d z d x & =\iiint_{\vec{r}_{G}} \frac{\partial}{\partial z^{*}} f(\vec{r}) d z^{*} d z d x, \\
-\iint_{\vec{r}_{g}}^{\cup} f(\vec{r}) d z^{*} d x & =\iiint_{\vec{r}_{G}} \frac{\partial}{\partial z} f(\vec{r}) d z^{*} d z d x, \\
\iint_{\vec{r}_{g}}^{\cup} f(\vec{r}) d z^{*} d z & =\iiint_{\vec{r}_{G}} \frac{\partial}{\partial \varkappa} f(\vec{r}) d z^{*} d z d x .
\end{aligned}
$$

Also, for a complex vector function $\vec{F}(\vec{r})=P(\vec{r}) \vec{w}_{1}+Q(\vec{r}) \vec{w}_{2}+R(\vec{r}) \vec{n}$ whose components are defined and continuous (integrable) functions possessing defined and continuous (integrable) partial derivatives on (over) the bounded domain $\vec{r}_{G}$ in the three-dimensional Euclidean complex vector space $\vec{r}$, it follows from previously derived results that

$$
\begin{aligned}
& \iint_{\vec{r}_{g}}^{\cup} \vec{F}(\vec{r}) \cdot d \vec{\sigma}=\iiint_{\vec{r}_{G}} \nabla \cdot \vec{F}(\vec{r}) d v, \\
& \iint_{\vec{r}_{g}}^{\cup} d \vec{\sigma} \times \vec{F}(\vec{r})=\iiint_{\vec{r}_{G}} \nabla \times \vec{F}(\vec{r}) d v .
\end{aligned}
$$

The integral equalities (2.11) and (2.12) correspond to that of the so-called Gauss-Ostrogradski theorem attached to the three-dimensional Euclidean real vector space $\vec{r}$, see [2].

In view of the fact that the limiting value $\vec{A}_{\vec{r}_{N}}$ of the sequence of reduced absolute integral sums $\vec{A}_{\Delta_{j_{1}, \ldots, j_{n}} \vec{r}_{g}}$ (see equality (2.12) of Definition 2.1) does not depend on the form of a contour surface $\vec{r}_{g}$ bounding the domain $\vec{r}_{G}$ in the three-dimensional Euclidean complex vector space $\vec{r}$, it follows that if $\vec{r}_{s}\left(\vec{r}_{N}, d \delta\right)$ is an infinitesimally small spherical surface centred at $\vec{r}_{N}$ and of radius $d \delta$, then for every point $\vec{r}_{N}$ lying inside $\vec{r}_{G}\left(\vec{r}_{N} \in \operatorname{int} \vec{r}_{G}\right.$, where int $\vec{r}_{G}$ is an interior of $\vec{r}_{G}$ ),

$$
\vec{A}_{\vec{r}_{N}}=\frac{1}{d_{\overrightarrow{r_{N}}} v} \iint_{\vec{r}_{S}\left(\vec{r}_{N}, d \delta\right)}^{\cup} f(\vec{r}) d \vec{\sigma} .
$$

For a point $\vec{r}_{N}$ on the boundary $\vec{r}_{g}$ of $\vec{r}_{G}\left(\vec{r}_{N} \in \vec{r}_{g}\right)$,

$$
\vec{A}_{\vec{r}_{N}}=\frac{1}{d_{\overrightarrow{r_{N}}} v} \iint_{\operatorname{int} \vec{r}_{s}\left(\vec{r}_{N}, d \delta\right)}^{\cup} f(\vec{r}) d \vec{\sigma},
$$


where int $\vec{r}_{s}\left(\vec{r}_{N}, d \delta\right)$ is a part of an infinitesimally small spherical surface $\vec{r}_{S}\left(\vec{r}_{N}\right.$, $d \delta$ ) laying inside $\vec{r}_{g}$.

Comment 2.2. Since the spatial derivative of a real-valued function $f(x)$ of the one variable $x$, which is defined on the segment $[a, b]$ of the real axis $R^{1}$, at a point $c$ lying inside $[a, b]$ is by definition

$$
\lim _{[a, b] \rightarrow c} \frac{f(b)-f(a)}{b-a}=A_{c},
$$

it follows from (2.13) that

$$
\begin{aligned}
A_{c} & =\frac{1}{d_{c} x}\left[f\left(c+0^{+}\right)-f\left(c-0^{+}\right)\right] \\
& =\lim _{2 \Delta x \rightarrow d_{c} x} \frac{f(c+\Delta x)-f(c-\Delta x)}{2 \Delta x} .
\end{aligned}
$$

Also, for boundary points of $[a, b]$, it follows from (2.14) that

$$
\begin{aligned}
& A_{a}=\frac{1}{d_{a} x}\left[f\left(a+0^{+}\right)-f(a)\right]=\lim _{\Delta x \rightarrow d_{a} x} \frac{f(a+\Delta x)-f(a)}{\Delta x}, \\
& A_{b}=\frac{1}{d_{b} x}\left[f(b)-f\left(b-0^{+}\right)\right]=\lim _{\Delta x \rightarrow d_{b} x} \frac{f(b)-f(b-\Delta x)}{\Delta x} .
\end{aligned}
$$

Accordingly, from (2.7), we obtain

$$
f(b)-f(a)=\sum_{c \in[a, b]} A_{c} d_{c} x
$$

If $A(x)\left(A(c)=A_{c}\right)$ is integrable over $[a, b]$, then $f(x)$ is continuous on $[a, b]$ and

$$
f(b)-f(a)=\int_{a}^{b} A(x) d x,
$$

where $A(x)=\nabla f(x)=d f(x) / d x$.

2.2. Residue of a complex function. From the functional equality (2.2) of Definition 2.1, more precisely, the integral relations (2.13) and (2.14), it follows that if $\vec{r}_{G}$ is a regular domain of a function $f(\vec{r})$, bounded by a contour surface $\vec{r}_{g}$ in the three-dimensional Euclidean complex vector space $\vec{r}$, then at any point $\vec{r}_{N}$ of $\vec{r}_{G}, \vec{A}_{\vec{r}_{N}} d_{\vec{r}_{N}} v=\overrightarrow{0}$.

On the other hand, if $\vec{r}_{G}$ is a singular domain of $f(\vec{r})$, then the absolute integral sum of the function $\vec{A}(\vec{r}), \sum_{\vec{r}_{N} \in \operatorname{int} \vec{r}_{G}} \vec{A}_{\vec{r}_{N}} d_{\vec{r}_{N}} v$, can be subdivided into two absolute integral sums:

$$
\sum_{\overrightarrow{r_{N}} \in \vec{r}_{G}} \vec{A}_{\vec{r}_{N}} d_{\vec{r}_{N}} v=\sum_{\overrightarrow{r_{N}} \in v p \vec{r}_{G}} \vec{A}_{\vec{r}_{N}} d_{\vec{r}_{N}} v+\sum_{\vec{r}_{N} \in v \vec{r}_{G}} \vec{A}_{\vec{r}_{N}} d_{\vec{r}_{N}} v,
$$


where $v p \vec{r}_{G}$ and $v s \vec{r}_{G}$ are sets of regular and singular points of the function $f(\vec{r})$ in the singular domain $\vec{r}_{G}$, respectively. Clearly, at all regular points of the singular domain of $f(\vec{r}), \vec{A}_{\vec{r}_{N}} d_{\vec{r}_{N}} v=\overrightarrow{0}$; in other words, the first absolute integral sum of $\vec{A}(\vec{r})$ on the left-hand side of the preceding relation is an infinite sum of zero vectors. At each singular point $\vec{r}_{N}$ of the domain $\vec{r}_{G}$, at which the sequence of the reduced absolute integral sum $\vec{A}_{\Delta_{j_{1}, \ldots, j_{n}} \vec{r}_{g}}$ definitely diverges, $\vec{A}_{\overrightarrow{r_{N}}} d_{\overrightarrow{r_{N}}} v$ reduces to an indefinite expression, more precisely, to either definite or indefinite vector value of the extended vector space $\vec{r} \cup \vec{r}_{\infty}$, where $\vec{r}_{\infty}$ is a set of infinite points.

2.2.1. A potential of a point with respect to a contour surface of integration. In the two-dimensional Euclidean real vector space $\vec{\varrho}=x \vec{e}_{1}+y \vec{e}_{2}$, an intensity of the vector $(\vec{\varrho} \times d \vec{\varrho}) / \vec{\varrho} \cdot \vec{\varrho}:(\vec{\varrho} \times d \vec{\varrho}) \cdot \vec{n} / \vec{\varrho} \cdot \vec{\varrho}=(x d y-y d x) /\left(x^{2}+\right.$ $\left.y^{2}\right)=d \arctan (y / x)$ defines an infinitesimal evolution of the vector $\vec{\varrho}_{o}$ of the unit length as a unit vector of a position vector of an arbitrary point in $\vec{\varrho}$ with respect to the origin

$$
\frac{(\vec{\varrho} \times d \vec{\varrho}) \cdot \vec{n}}{\vec{\varrho} \cdot \vec{\varrho}}=\left|d \vec{\varrho}_{o}\right|=d \varphi .
$$

In the two-dimensional Euclidean complex vector space $\vec{\varrho}=|\vec{\varrho}| \vec{\varrho}_{o}$, it holds that

$$
\int_{\vec{\varrho}_{g}}^{\cup} \frac{(\vec{\varrho} \times d \vec{\varrho}) \cdot \vec{n}}{\vec{\varrho} \cdot \vec{\varrho}^{*}}=\int_{\vec{\varrho}_{g}}^{\cup}\left(\vec{\varrho}_{o} \times d \vec{\varrho}_{o}\right) \cdot \vec{n}=i \int_{\vec{\varrho}_{g}}^{\cup} d \varphi .
$$

Since $e^{2 i \arctan (y / x)}=(1+i(y / x))^{2} /\left(1+(y / x)^{2}\right)=(x+i y) /(x-i y)$, it follows from the functional equality $\varphi=\arctan (y / x)$ that $\vec{\varrho}=\left(\sqrt{\vec{\varrho} \cdot \vec{\varrho}^{*} / 2}\right)\left(e^{-i \varphi} \vec{w}_{1}+\right.$ $\left.e^{i \varphi} \vec{w}_{2}\right)=|\vec{\varrho}| \vec{\varrho}_{o}$, where $|\vec{\varrho}|=\sqrt{\vec{\varrho} \cdot \vec{\varrho}^{*}}$ and $\left.\vec{\varrho}_{o}=(1 / \sqrt{2})\left(e^{-i \varphi} \vec{w}_{1}+e^{i \varphi} \vec{w}_{2}\right)\right)$

DEFINITION 2.3. A potential $p_{\vec{\varrho}_{g} \rightarrow \vec{\varrho}_{N}}$ of a point $\vec{\varrho}_{N}$ with respect to a contour $\vec{\varrho}_{g}$ bounding an arbitrary domain $\vec{\varrho}_{G}$ in the two-dimensional Euclidean complex vector space $\vec{\varrho}$ is by definition

$$
p_{\vec{\varrho}_{g} \rightarrow \vec{\varrho}_{N}}=\int_{\vec{\varrho}_{g}}^{\cup} \frac{\left[\left(\vec{\varrho}-\vec{\varrho}_{N}\right) \times d \vec{\varrho}\right] \cdot \vec{n}}{\left(\vec{\varrho}-\vec{\varrho}_{N}\right) \cdot\left(\vec{\varrho}-\vec{\varrho}_{N}\right)^{*}} .
$$

In view of the fact that differential $d \varphi$ is an absolute one for any closed path of integration $\vec{\varrho}_{g}$ bounding the domain $\vec{\varrho}_{G}$ in the complex plane $\vec{\varrho}$, it follows that

$$
p_{\vec{\varrho}_{g} \rightarrow \vec{\varrho}_{N}}=i \int_{\vec{\varrho}_{g}}^{\cup} d \theta= \begin{cases}2 \pi i & \text { for } \vec{\varrho}_{N} \in \operatorname{int} \vec{\varrho}_{G}, \\ 0 & \text { for } \vec{\varrho}_{N} \notin \vec{\varrho}_{G},\end{cases}
$$

where int $\vec{\varrho}_{G}$ is an interior of $\vec{\varrho}_{G}$. 
In the case when a point $\vec{\varrho}_{N}$ belongs to the boundary $\vec{\varrho}_{g}$ of $\vec{\varrho}_{G}$, the potential $p_{\vec{\varrho}_{g} \rightarrow \vec{\varrho}_{N}}$ of a point $\vec{\varrho}_{N}$ with respect to $\vec{\varrho}_{g}$ is defined to be the sum of limiting values of $i \int_{\vec{\varrho}_{g}}^{U} d \theta$ over a part of the path of integration $\vec{\varrho}_{g}$ from the point $\vec{\varrho}_{A}$ to the point $\vec{\varrho}_{B}\left(\vec{\varrho}_{A}\right.$ and $\vec{\varrho}_{B}$ are intersection points of the path of integration $\vec{\varrho}_{g}$ and some arbitrary small circle $\vec{\varrho}_{\delta}$ centred at $\vec{\varrho}_{N}$ and of radius $\delta$ ) as well as over circular arcs from the point $\vec{\varrho}_{B}$ to the point $\vec{\varrho}_{A}$, when the radius $\delta$ of $\vec{\varrho}_{\delta}$ tends to zero, in other words, when the boundary points of the circular arcs $\vec{\varrho}_{A}$ and $\vec{\varrho}_{B}$, along the path of integration $\vec{\varrho}_{g}$, tend to the point $\vec{\varrho}_{N}$.

Considering the fact that the limiting value of $i \int_{\vec{\varrho}_{g}}^{u} d \theta$ over a part of the path of integration $\vec{\varrho}_{g}$ from the point $\vec{\varrho}_{A}$ to the point $\vec{\varrho}_{B}$ is equal to

$$
\lim _{\delta \rightarrow 0^{+}} i \int_{\vec{\varrho}_{g}}^{\stackrel{\vec{\varrho}_{B} \vec{\varrho}_{A}}{\vec{Q}_{g}}} d \theta=i \alpha, \quad \lim _{\delta \rightarrow 0^{+}} i \int_{\operatorname{int} \vec{\varrho}_{\delta}}^{\stackrel{\vec{\varrho}_{B} \vec{\varrho}_{A}}{S}} d \theta=-i \alpha,
$$

as well as

$$
\lim _{\delta \rightarrow 0^{+}} i \int_{\operatorname{ext} \hat{\varrho}_{\delta}}^{\vec{\varrho}_{A} \vec{\varrho}_{B}} d \theta=i(2 \pi-\alpha)
$$

where int $\vec{\varrho}_{\delta}$ and ext $\vec{\varrho}_{\delta}$ are circular arcs inside and outside $\vec{\varrho}_{g}$, respectively, and $\alpha$ is a limit angle of tangent lines to $\vec{\varrho}_{g}$ at the points $\vec{\varrho}_{A}$ and $\vec{\varrho}_{B}$ in the case when the boundary points $\vec{\varrho}_{A}$ and $\vec{\varrho}_{B}$, along $\vec{\varrho}_{g}$, tend to the point $\vec{\varrho}_{N}$, it follows that

$$
p_{\vec{\varrho}_{g} \rightarrow \vec{\varrho}_{N}}=\left\{\begin{array}{l}
0, \\
2 \pi i .
\end{array}\right.
$$

DEFINITION 2.4. A potential $p_{\vec{r}_{y} \rightarrow \vec{r}_{N}}$ of a point $\vec{r}_{N}$ with respect to a contour surface $\vec{r}_{g}$ bounding the domain $\vec{r}_{G}$ in the three-dimensional Euclidean complex vector space $\vec{r}=\vec{\varrho}+\varkappa \vec{n}$ is by definition

$$
p_{\vec{r}_{g} \rightarrow \vec{r}_{N}}=2 p_{\varrho_{g} \rightarrow \vec{\varrho}_{N}},
$$

where $\vec{\varrho}_{g}=\vec{r}_{g} \cap \vec{\varrho}$ and $\vec{\varrho}$ is any complex plane such that $\vec{r}_{N} \equiv \vec{\varrho}_{N}$.

If one takes into consideration the fact that except the point $\vec{r}_{N}$, which is an inner point with respect to an infinitesimally small spherical surface $\vec{r}_{s}\left(\vec{r}_{N}, d \delta\right)$, all remaining points of the vector space $\vec{r}$ are external points, then according to the defined notion of the potential $p_{\vec{r}_{g} \rightarrow \vec{r}_{N}}$ of a point $\vec{r}_{N}$ with respect to a contour surface $\vec{r}_{g}$ bounding a certain domain $\vec{r}_{G}$ in $\vec{r}$ and Poor's definition of nonanalytic functions residue (see [3, Definition 1, page 38]), the notion of residues of a scalar-valued function $f(\vec{r})$ can be defined as follows. 
DEFINITION 2.5. A residue (Res) of a scalar-valued function $f(\vec{r})$ at a point $\vec{r}_{N}$ in the three-dimensional Euclidean complex vector space $\vec{r}$ is by definition

$$
\iint_{\vec{r}_{S}\left(\vec{r}_{N}, d \delta\right)}^{\cup} f(\vec{r}) d \vec{\sigma}=p_{\vec{r}_{S}\left(\overrightarrow{r_{N}}, d \delta\right) \rightarrow \overrightarrow{r_{N}}} \underset{\vec{r}=\vec{r}_{N}}{\operatorname{Res}} f(\vec{r}) .
$$

DEFINITION 2.6. A residue (Res) of a scalar-valued function $f(\vec{r})$ at the set of the infinite points $\vec{r}_{\infty}$ is by definition

$$
\underset{\vec{r}=\vec{r}_{\infty}}{\overrightarrow{\operatorname{ras}_{\infty}}} f(\vec{r})=-\sum_{\vec{r}_{N} \in \vec{r}} \underset{\vec{r}=\overrightarrow{r_{N}}}{\overrightarrow{\operatorname{Res}}} f(\vec{r}) \text {. }
$$

From equality (2.29) of Definition 2.5, it follows that at all points inside a certain singular domain $\vec{r}_{G}$ bounded by a contour surface $\vec{r}_{g}\left(\vec{r}_{N} \in \operatorname{int} \vec{r}_{G}\right)$,

$$
\vec{A}_{\vec{r}_{N}} d_{\vec{r}_{N}} v=\nabla_{\vec{r}_{N}} f(\vec{r}) d_{\vec{r}_{N}} v=p_{\vec{r}_{g} \rightarrow \vec{r}_{N}} \underset{\vec{r}=\vec{r}_{N}}{\overrightarrow{\operatorname{Res}}} f(\vec{r}),
$$

while at points on the boundary $\vec{r}_{g}$ of $\vec{r}_{G}\left(\vec{r}_{N} \in \vec{r}_{g}\right)$,

$$
\vec{A}_{\vec{r}_{N}} d_{\vec{r}_{N}} v=p_{\operatorname{int} \vec{r}_{s}\left(\vec{r}_{N}, d \delta\right) \rightarrow \overrightarrow{r_{N}}} \underset{\vec{r}=\vec{r}_{N}}{\overrightarrow{\operatorname{Res}}} f(\vec{r}),
$$

where according to equality (2.28), $p_{\operatorname{int} \vec{r}_{S}\left(\vec{r}_{N}, d \delta\right) \rightarrow \vec{r}_{N}}=2 p_{\text {int } \vec{\varrho}_{S}\left(\vec{\varrho}_{N}, d \delta\right) \rightarrow \vec{\varrho}_{N}}$ and

$$
p_{\operatorname{int} \vec{\varrho}_{S}\left(\vec{\varrho}_{N}, d \delta\right) \rightarrow \vec{\varrho}_{N}}=i \int_{\operatorname{int} \vec{\varrho}_{S}\left(\vec{\varrho}_{N}, d \delta\right)}^{\curvearrowleft} d \theta=\lim _{\delta \rightarrow 0^{+}} i \int_{\operatorname{int} \vec{\varrho}_{\delta}}^{\vec{\varrho}_{B} \vec{\varrho}_{A}} d \theta=i \alpha,
$$

and $\alpha$ is an angle of tangent lines at the point $\vec{\varrho}_{N}$ lying on the boundary $\vec{\varrho}_{g}$ of the domain $\vec{\varrho}_{G}: \vec{\varrho}_{G}=\vec{r}_{G} \cap \vec{\varrho}\left(\vec{\varrho}_{g}=\vec{r}_{g} \cap \vec{\varrho}\right)$.

Clearly,

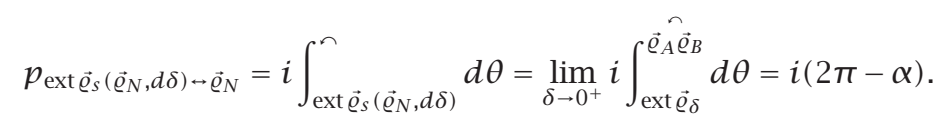

DEFINITION 2.7. Cauchy's principal value $(v p)$ of an improper integral of a vector-valued function $\nabla f(\vec{r})$, with respect to a certain domain $\vec{r}_{G}$ bounded by a contour surface $\vec{r}_{g}$ in the three-dimensional Euclidean complex vector space $\vec{r}$, is by definition

$$
v p \iiint_{\vec{r}_{G}} \nabla f(\vec{r}) d v=\sum_{\vec{r}_{N} \in v p \vec{r}_{G}} \vec{A}_{\vec{r}_{N}} d_{\vec{r}_{N}} v
$$

where $v p \vec{r}_{G}$ is a set of regular points $\vec{r}_{N}$ of the function in the domain $\vec{r}_{G}$.

DEFINITION 2.8. Jordan's singular value $(v s)$ of an improper integral of a vector-valued function $\nabla f(\vec{r})$, with respect to a certain domain $\vec{r}_{G}$ bounded by 
a contour surface $\vec{r}_{g}$ in the three-dimensional Euclidean complex vector space $\vec{r}$, is by definition

$$
v s \iiint_{\vec{r}_{G}} \nabla f(\vec{r}) d v=\sum_{\vec{r}_{N} \in v s \vec{r}_{G}} \vec{A}_{\vec{r}_{N}} d_{\vec{r}_{N}} v
$$

where $v s \vec{r}_{G}$ is a set of singular points $\vec{r}_{N}$ of the function in the domain $\vec{r}_{G}$.

Definition 2.9. The sum of Cauchy's principal value $(v p)$ and Jordan's singular value $(v s)$ is a total value $(v t)$ of an improper integral.

Since the derived equality (2.6) holds also in the case when the sequences of reduced absolute integral sums $\vec{A}_{\dot{j}_{1}, \ldots, j_{n} \vec{r}_{g}}$ diverge, in other words, in the case of a singular domain $\vec{r}_{G}$ of the function $f(\vec{r})$, it follows that if $f(\vec{r})$ is an integrable function over a contour surface $\vec{r}_{g}$ bounding a certain singular domain $\vec{r}_{G}$ of $f(\vec{r})$, then

$$
\iint_{\overrightarrow{r_{g}}}^{\cup} f(\vec{r}) d \vec{\sigma}=\sum_{\overrightarrow{r_{N}} \in v p \vec{r}_{G}} \vec{A}_{\vec{r}_{N}} d_{\vec{r}_{N}} v+\sum_{\overrightarrow{r_{N}} \in v s \vec{r}_{G}} \vec{A}_{\vec{r}_{N}} d_{\vec{r}_{N}} v .
$$

Finally, on the basis of Definition 2.9 as well as equalities (2.35) and (2.36), we obtain

$$
\iint_{\vec{r}_{g}}^{\cup} f(\vec{r}) d \vec{\sigma}-v p \iiint_{\vec{r}_{G}} \nabla f(\vec{r}) d v=\sum_{\vec{r}_{N} \in v s \vec{r}_{G}} p_{\vec{r}_{g} \rightarrow \vec{r}_{N}} \overrightarrow{\operatorname{Res}_{\vec{r}}=\vec{r}_{N}} f(\vec{r}),
$$

where $v s \vec{r}_{G}$ is a set of singular points $\vec{r}_{N}$ of $f(\vec{r})$ in the singular domain $\vec{r}_{G}$ bounded by a contour surface $\vec{r}_{g}$ in the three-dimensional Euclidean complex vector space $\vec{r}$, more precisely

$$
\iint_{\vec{r}_{g}}^{\cup} f(\vec{r}) d \vec{\sigma}=v t \iiint_{\vec{r}_{G}} \nabla f(\vec{r}) d v
$$

If a function $f(\vec{r})$ is not integrable over a contour surface $\vec{r}_{g}$ bounding a certain singular domain $\vec{r}_{G}$ of the function, in other words, if singularities of the function $f(\vec{r})$ lie not only inside but on the contour surface $\vec{r}_{g}$ too, then on the one hand

$$
\begin{array}{r}
\sum_{\overrightarrow{r_{N}} \in v p \vec{r}_{g}} f\left(\vec{r}_{N}\right) d_{\vec{r}_{N}} \vec{\sigma}+\sum_{\vec{r}_{N} \in v s \vec{r}_{y}} \iint_{\operatorname{int} \vec{r}_{S}\left(\vec{r}_{N}, d \delta\right)}^{\curvearrowright} f(\vec{r}) d \vec{\sigma} \\
=v p \iiint_{\vec{r}_{G}} \nabla f(\vec{r}) d v+\sum_{\vec{r}_{N} \in v s \operatorname{int} \vec{r}_{G}} 4 \pi \underset{\vec{r}=\vec{r}_{N}}{\operatorname{Res}} f(\vec{r}),
\end{array}
$$


and on the other

$$
\begin{array}{r}
\sum_{\vec{r}_{N} \in v p \vec{r}_{g}} f\left(\vec{r}_{N}\right) d_{\vec{r}_{N}} \vec{\sigma}+\sum_{\vec{r}_{N} \in v s \vec{r}_{\gamma}} \iint_{\operatorname{ext} \vec{r}_{s}\left(\vec{r}_{N}, d \delta\right)}^{n} f(\vec{r}) d \vec{\sigma} \\
=v p \iiint_{\vec{r}_{G}} \nabla f(\vec{r}) d v+\sum_{\overrightarrow{r_{N}} \in v \vec{r}_{G}} 4 \pi i \underset{\vec{r}=\overrightarrow{r_{N}}}{\operatorname{Res}} f(\vec{r}),
\end{array}
$$

more precisely

$$
v t \iint_{\vec{r}_{g}}^{\cup} f(\vec{r}) d \vec{\sigma}-v p \iiint_{\vec{r}_{G}} \nabla f(\vec{r}) d v=\sum_{\vec{r}_{N} \in v s \vec{r}_{G}} p_{\vec{r}_{g} \rightarrow \vec{r}_{N}} \underset{\vec{r}=\vec{r}_{N}}{\overrightarrow{\operatorname{Res}}} f(\vec{r}),
$$

where

$$
\begin{aligned}
v t \iint_{\overrightarrow{r_{g}}}^{\cup} f(\vec{r}) d \vec{\sigma} & =v p \iint_{\vec{r}_{g}}^{\cup} f(\vec{r}) d \vec{\sigma}+v s \iint_{\vec{r}_{g}}^{\cup} f(\vec{r}) d \vec{\sigma} \\
& =\sum_{\overrightarrow{r_{N}} \in v p \vec{r}_{g}} f\left(\vec{r}_{N}\right) d_{\overrightarrow{r_{N}}} \vec{\sigma}+\sum_{\vec{r}_{N} \in v s \vec{r}_{g}}\left\{\begin{array}{c}
-p_{\text {int }} \cdot \vec{r}_{S}\left(\vec{r}_{N}, d \delta\right) \rightarrow \vec{r}_{N} \\
p_{\operatorname{ext}} \cdot \vec{r}_{s}\left(\vec{r}_{N}, d \delta\right) \rightarrow \vec{r}_{N}
\end{array}\right\} \underset{\vec{r}=\vec{r}_{N}}{\operatorname{Res}} f(\vec{r}) .
\end{aligned}
$$

COMMENT 2.10. For a real-valued function $f(x)$ of the one variable $x$, which is spatially differentiable almost everywhere over the segment $[a, b]$ of the real axis $R^{1}$ and defined at boundary points $a$ and $b$ of $[a, b]$, it follows from (2.38) that

$$
f(b)-f(a)-v p \int_{a}^{b} \nabla f(x) d x=\sum_{c \in v s[a, b]} p_{a, b \rightarrow c} \operatorname{Res}_{x=c} f(x),
$$

where $2 p_{a, b \rightarrow c}=p_{\vec{\varrho}_{g} \rightarrow \vec{\varrho}_{c}}$ and $\vec{\varrho}_{g}: \vec{\varrho}_{g} \cap R^{1}=\{a, b\}$ as well as

$$
p_{a, b \rightarrow c} \operatorname{Res}_{x=c} f(x)=f\left(c+0^{+}\right)-f\left(c-0^{+}\right)=A_{c} d_{c} x
$$

more precisely

$$
p_{a, b \rightarrow c} \operatorname{Res}_{x=c} f(x)=\lim _{2 \Delta x \rightarrow d_{c} x}[f(c+\Delta x)-f(c-\Delta x)] .
$$

Based on Definitions 2.7, 2.8, and 2.9,

$$
v t \int_{a}^{b} \nabla f(x) d x=f(b)-f(a)
$$

EXAMPLE 2.11. The scalar-valued function $f(x)=\log x$, where $\log$ denotes principal logarithm, is spatially differentiable at all points of the segment 
$[-a, b]$ of the real axis $R^{1}\left(a, b \in R_{+}^{1}\right)$ except at the point $x=0$. Since

$$
\begin{gathered}
p_{-a, b \rightarrow 0} \operatorname{Res}_{x=0} f(x)=\lim _{2 \Delta x \rightarrow d_{0} x}[\log (\Delta x)-\log (-\Delta x)]=\mp \pi i, \\
v p \int_{-a}^{b} \nabla f(x) d x=\log \frac{b}{a},
\end{gathered}
$$

it follows that $v t \int_{-a}^{b}(d x / x)=\log (b / a) \mp \pi i$.

EXAMPLE 2.12. The scalar-valued function $f(x)=x^{-1}$ is spatially differentiable at all points of the segment $[-a, b]$ of the real axis $R^{1}\left(a, b \in R_{+}^{1}\right)$ except at the point $x=0$. Since

$$
\begin{gathered}
p_{-a, b \rightarrow 0} \operatorname{Res}_{x=0} f(x)=\lim _{2 \Delta x \rightarrow d_{0} x}\left[\frac{1}{\Delta x}+\frac{1}{\Delta x}\right]=+\infty, \\
v p \int_{-a}^{b} \nabla f(x) d x=-\infty,
\end{gathered}
$$

in this case the total value of an improper integral $v t \int_{-a}^{b} x^{-2} d x$, as an indefinite expression $\infty-\infty$, has exactly definite value

$$
v t \int_{-a}^{b} \frac{d x}{x^{2}}=-\frac{b+a}{a b} .
$$

Let the singular domain $\vec{r}_{G}$ of the function $f(\vec{r}): f(\vec{r})=f\left(z^{*}, z\right)$, defined on the complex plane $\vec{\varrho}$, be a cylindrical domain bounded by a contour surface $\vec{r}_{g}$ in the three-dimensional Euclidean complex vector space $\vec{r}: \vec{r}=\vec{\varrho}+\varkappa \vec{n}$, whose bases are obtained by translation of the domain $\vec{\varrho}_{G}$ bounded by a contour $\vec{\varrho}_{g}$ in the complex plane $\vec{\varrho}$ in the direction of the unit normal vector $\vec{n}$ for constant values $-h$ and $h\left(\varkappa_{1}(\vec{\varrho})=-h\right.$ and $\left.\varkappa_{2}(\vec{\varrho})=h\right)$. In this case, if the function $f(\vec{r})$ is integrable over the contour of integration $\vec{\varrho}_{g}$, then it follows from (2.38) that

$$
\begin{aligned}
& \int_{\vec{\varrho}_{g}}^{\cup} f\left(z, z^{*}\right) d z-v p \iint_{\vec{\varrho}_{G}} \frac{\partial}{\partial z^{*}} f\left(z, z^{*}\right) d z^{*} d z \\
& =\sum_{\vec{\varrho}_{N} \in v s \vec{\varrho}_{G}} p_{\vec{\varrho}_{g} \rightarrow \vec{\varrho}_{N}} \operatorname{Res}_{\vec{\varrho}=\vec{\varrho}_{N}} f\left(z, z^{*}\right), \\
& -\int_{\vec{\varrho}_{g}}^{\cup} f\left(z, z^{*}\right) d z^{*}-v p \iint_{\vec{\varrho}_{G}} \frac{\partial}{\partial z} f\left(z, z^{*}\right) d z^{*} d z \\
& =\sum_{\vec{\varrho}_{N} \in v s \vec{\varrho}_{G}} p_{\vec{\varrho}_{g} \rightarrow \vec{\varrho}_{N}} \operatorname{Res}_{\vec{\varrho}=\vec{\varrho}_{N}}^{\star} f\left(z, z^{*}\right) .
\end{aligned}
$$

Clearly, partial residues of the function $f\left(z, z^{*}\right)$ are by definition

$$
\begin{aligned}
& \int_{\vec{\varrho}_{S}\left(\vec{\varrho}_{N}, d \delta\right)}^{\cup} f\left(z, z^{*}\right) d z=p_{\vec{\varrho}_{S}\left(\vec{\varrho}_{N}, d \delta\right) \rightarrow \vec{\varrho}_{N}} \operatorname{Res}_{\vec{\varrho}=\underline{\varrho}_{N}} f\left(z, z^{*}\right), \\
& -\int_{\vec{\varrho}_{S}\left(\vec{\varrho}_{N}, d \delta\right)}^{\cup} f\left(z, z^{*}\right) d z^{*}=p_{\vec{\varrho}_{s}\left(\vec{\varrho}_{N}, d \delta\right) \oplus \vec{\varrho}_{N}} \underset{\vec{\varrho}_{\hat{\varrho}}=\vec{\varrho}_{N}}{\stackrel{\star}{\operatorname{Res}} f} f\left(z, z^{*}\right) .
\end{aligned}
$$


DEFINITION 2.13. The function $f(\vec{r})$ is a regular-analytic function on the domain $\vec{\varrho}_{G}$ in the complex plane $\vec{\varrho}$, which is a regular domain of $f(\vec{r})$, if and only if a function $f(\vec{\varrho})=f\left(z, z^{*}\right)$, at any point $\vec{\varrho}_{N}$ of $\vec{\varrho}_{G}$, satisfies one of the conditions $\left\{\left(\partial / \partial z^{*}\right) f\left(z, z^{*}\right)\right\}_{\varrho_{N}}=0$ or $\left\{(\partial / \partial z) f\left(z, z^{*}\right)\right\}_{\vec{\varrho}_{N}}=0$.

DEFINITION 2.14. The function $f(\vec{r})$ is a singular-analytic function on the domain $\vec{\varrho}_{G}$ in the complex plane $\vec{\varrho}$, which is a singular domain of $f(\vec{r})$, if and only if a function $f(\vec{\varrho})=f\left(z, z^{*}\right)$, at any point $\vec{\varrho}_{N}$ of $\vec{\varrho}_{G}$, satisfies one of the conditions: $\left\{\left(\partial / \partial z^{*}\right) f\left(z, z^{*}\right)\right\}_{\hat{\varrho}_{N}}=0$ or $\left\{(\partial / \partial z) f\left(z, z^{*}\right)\right\}_{\hat{\varrho}_{N}}=0$.

For a complex vector-valued function $\vec{F}(\vec{\varrho})=P(\underline{\varrho}) \vec{w}_{1}+Q(\vec{\varrho}) \vec{w}_{2}$ defined on the two-dimensional Euclidean complex vector space $\vec{\varrho}$, whose components $P(\vec{\varrho})$ and $Q(\vec{\varrho})$ are integrable functions over a contour of integration $\vec{\varrho}_{g}$ bounding a singular domain $\vec{\varrho}_{G}$ of $\vec{F}(\vec{\varrho})$,

$$
\begin{aligned}
& \int_{\vec{\varrho}_{g}}^{\cup}[\vec{F}(\vec{\varrho}) \times d \vec{\varrho}] \cdot \vec{n}-v p \iint_{\vec{\varrho}_{G}}[\nabla \cdot \vec{F}(\vec{\varrho})](d \vec{\sigma} \cdot \vec{n}) \\
& =\sum_{\vec{\varrho}_{N} \in v s \vec{\varrho}_{G}} p_{\vec{\varrho}_{g} \rightarrow \vec{\varrho}_{N}} \underset{\vec{\varrho}=\vec{\varrho}_{N}}{\operatorname{Res}} \vec{F}(\vec{\varrho}), \\
& \int_{\vec{\varrho}_{g}}^{\cup} \vec{F}(\vec{\varrho}) \cdot d \vec{\varrho}-v p \iint_{\hat{\varrho}_{G}} \vec{n} \cdot[\nabla \times \vec{F}(\vec{\varrho})](d \vec{\sigma} \cdot \vec{n}) \\
& =\sum_{\vec{\varrho}_{N} \in v s \vec{\varrho}_{G}} p_{\vec{\varrho}_{g} \rightarrow \vec{\varrho}_{N}} \underset{\vec{\varrho}=\vec{\varrho}_{N}}{\stackrel{\star}{\operatorname{Re}}} \vec{F}(\vec{\varrho}) .
\end{aligned}
$$

In this case

$$
\begin{aligned}
& \operatorname{Res}_{\vec{\varrho}=\vec{\varrho}_{N}} \vec{F}(\vec{\varrho})=\operatorname{Res}_{\vec{\varrho}=\vec{\varrho}_{N}} P\left(z, z^{*}\right)+\operatorname{Res}_{\vec{\varrho}=\vec{\varrho}_{N}}^{\star} Q\left(z, z^{*}\right) \text {, }
\end{aligned}
$$

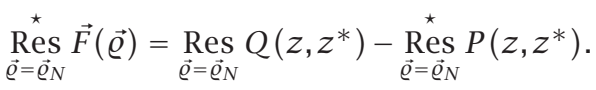

EXAMPLE 2.15. A domain $\vec{\varrho}_{G_{\delta}}=\left\{\vec{\varrho}: a \geq|\vec{\varrho}| \geq \delta ;(\delta, a) \in R_{+}^{1}\right\}$ of the complex plane $\vec{\varrho}$ is a regular domain of the function $\left(z, z^{*}\right) \mapsto(1 / 2) \log \left(z z^{*}\right)$. Taking into account the fact that $(1 / 2) \log \left(z z^{*}\right)=(1 / 2) \ln \left(x^{2}+y^{2}\right)$, it follows from the result of the well-known Green-Riemann theorem that

$$
\begin{gathered}
\int_{\vec{\varrho}_{a}}^{\cup} \log \left(z z^{*}\right) d z-\int_{\vec{\varrho}_{\delta}}^{\cup} \log \left(z z^{*}\right) d z \\
=2 i \iint_{\vec{\varrho}_{G_{\delta}}} \frac{x+i y}{x^{2}+y^{2}} d x d y, \\
-\int_{\vec{\varrho}_{a}}^{\cup} \log \left(z z^{*}\right) d z^{*}+\int_{\vec{\varrho}_{\delta}}^{\cup} \log \left(z z^{*}\right) d z^{*} \\
\quad=2 i \iint_{\vec{\varrho}_{G_{\delta}}} \frac{x-i y}{x^{2}+y^{2}} d x d y .
\end{gathered}
$$


Similarly, as $(1 / 2) \log \left(z / z^{*}\right)=i \arctan (y / x)$, then

$$
\begin{aligned}
& \int_{\vec{\varrho}_{a}}^{\stackrel{n}{\vec{\varrho}_{B} \vec{\varrho}_{A}}} \log \frac{z}{z^{*}} d z+\int_{\vec{\varrho}_{1}}^{\stackrel{\vec{\varrho}_{B}}{\overrightarrow{\varrho_{C}}}} \log \frac{z}{z^{*}} d z+\int_{\vec{\varrho}_{\delta}}^{\stackrel{\vec{\varrho}_{C}}{\vec{\varrho}_{D}}} \log \frac{z}{z^{*}} d z+\int_{\vec{\varrho}_{2}}^{\stackrel{\vec{\varrho}_{D}}{\vec{\varrho}_{A}}} \log \frac{z}{z^{*}} d z \\
& =-2 i \iint_{\vec{\varrho}_{G_{\delta}} \backslash \triangleright \vec{\varrho}_{G_{\delta}}} \frac{x+i y}{x^{2}+y^{2}} d x d y,
\end{aligned}
$$

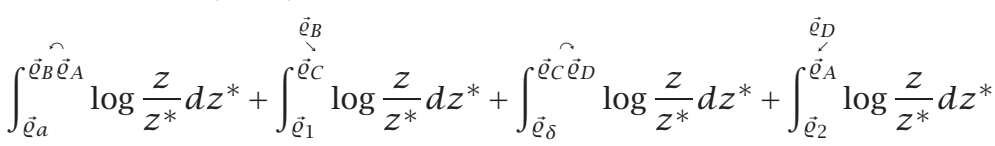

$$
\begin{aligned}
& =-2 i \iint_{\vec{\varrho}_{G_{\delta}} \backslash \triangleright \vec{\varrho}_{G_{\delta}}} \frac{x-i y}{x^{2}+y^{2}} d x d y,
\end{aligned}
$$

where the domain $\vec{\varrho}_{G_{\delta}} \backslash \triangleright \vec{\varrho}_{G_{\delta}}$ is a part of the domain $\vec{\varrho}_{G_{\delta}}$ bounded by parts of circular contours of integration $\vec{\varrho}_{a}$ and $\vec{\varrho}_{\delta}$ bounding $\vec{\varrho}_{G_{\delta}}$ also by segments of straight lines $\vec{\varrho}_{k}$ of $\vec{\varrho}: \vec{\varrho}_{k}=\left\{\vec{\varrho}: \vec{\varrho}=|\vec{\varrho}| \vec{\varrho}_{o k}\left(\varphi=\varphi_{k}\right)\right\}(k=1,2)$. The points $\vec{\varrho}_{A}, \vec{\varrho}_{B}, \vec{\varrho}_{C}$, and $\vec{\varrho}_{D}$ are obtained by an intersection of circular contours of integration $\vec{\varrho}_{a}$ and $\vec{\varrho}_{\delta}$ with directions $\vec{\varrho}_{k}$.

For arbitrary chosen angular values $\varphi_{k}$, when $\varphi_{1} \rightarrow \pi$ and $\varphi_{2} \rightarrow-\pi$, it follows that

$$
\begin{aligned}
& \int_{\vec{\varrho} a}^{\cup} \log \frac{z}{z^{*}} d z-\int_{\vec{\varrho}_{\delta}}^{\cup} \log \frac{z}{z^{*}} d z+\int_{\vec{\varrho}_{k}}^{\overrightarrow{ }} \log \frac{z}{z^{*}} d z \\
& \quad=-2 i \iint_{\vec{\varrho}_{G_{\delta}}} \frac{x+i y}{x^{2}+y^{2}} d x d y, \\
& \int_{\vec{\varrho}_{a}}^{\cup} \log \frac{z}{z^{*}} d z^{*}-\int_{\vec{\varrho}_{\delta}}^{\cup} \log \frac{z}{z^{*}} d z^{*}+\int_{\vec{\varrho}_{k}}^{\overrightarrow{ }} \log \frac{z}{z^{*}} d z^{*} \\
& \quad=-2 i \iint_{\vec{\varrho}_{G_{\delta}}} \frac{x-i y}{x^{2}+y^{2}} d x d y .
\end{aligned}
$$

In other words, the scalar-valued function $z \mapsto \log z$ :

$$
\log z=\frac{1}{2}\left[\log \left(z z^{*}\right)+\log \frac{z}{z^{*}}\right]
$$

is a singular-analytic one on the domain $\vec{\varrho}_{g}=\{\vec{\varrho}:|\vec{\varrho}| \leq a\}$ in the complex plane $\vec{\varrho}$, more precisely

$$
\begin{gathered}
\int_{\overrightarrow{\varrho_{a}}}^{\cup} \log z d z+\int_{\vec{\varrho}_{k}}^{\vec{\rightleftharpoons}} \log z d z=\lim _{\delta \rightarrow 0^{+}} \int_{\vec{\varrho}_{\delta}}^{\cup} \log z d z, \\
-\int_{\vec{\varrho}_{a}}^{\cup} \log z d z^{*}-v p \iint_{\vec{\varrho}_{g}} \frac{1}{z} d z d z^{*}-\int_{\vec{\varrho}_{k}}^{\rightleftharpoons} \log z d z^{*}=-\lim _{\delta \rightarrow 0^{+}} \int_{\vec{\varrho}_{\delta}}^{\cup} \log z d z^{*} .
\end{gathered}
$$


3. Conclusion. On the basis of a notion of absolute integral sums of a complex function, which is slightly more general with respect to that of integral sums of ordinary integral calculus, result (2.6) has been derived as an immediate consequence of equality (2.1) of Definition 2.1 so that its generality is undeniable since it is not conditioned by convergence of reduced absolute integral sums. In other words, the aforementioned result (2.6) is more general with respect to that of the well-known Cauchy fundamental theorem on residues of Cauchy's calculus of residues. Also, results (2.38) and (2.42) as well as the results of Section 2.2, which are based on the redefined notion of a complex function residue as well as the defined notion of the total value of an improper integral of a function with respect to a certain singular domain bounded by a contour surface in the three-dimensional Euclidean complex vector space and which are more general with respect to the fundamental results of Cauchy's calculus of residues of both analytic and nonanalytic functions, have been derived.

Any further generalization of the results of Cauchy's calculus of residues as well as of the results in near relation to other areas of either the pure or applied mathematics must have the aforementioned results as its base.

\section{REFERENCES}

[1] R. Bellman and K. L. Kuk, Differential-Difference Equations, Izdat. Mir, Moscow, 1967 (Russian).

[2] D. Mihailović and D. Đ. Tošić, Elements of Mathematical Analysis, Naučna Knjiga, Belgrade, 1983 (Serbian).

[3] D. S. Mitrinović and J. D. Kečkić, Cauchy's Calculus of Residues with Applications, Matematički Problemi i Ekspozicije. 8., Naučna Knjiga, Belgrade, 1978 (Serbo-Croatian).

[4] V. C. Poor, Residues of polygenic functions, Trans. Amer. Math. Soc. 32 (1930), no. 2, 216-222.

[5] _ _ On residues of polygenic functions, Trans. Amer. Math. Soc. 75 (1953), 244255.

[6] B. Saric, On the finite Fourier transforms of functions with infinite discontinuities, Int. J. Math. Math. Sci. 30 (2002), no. 5, 301-317.

Branko Sarić: 32000 Čačak, Kralja Petra I br.1, Serbia and Montenegro

E-mail address: bsaric@ptt.yu 


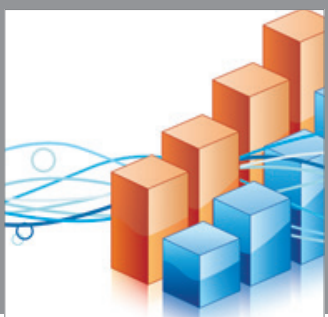

Advances in

Operations Research

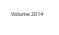

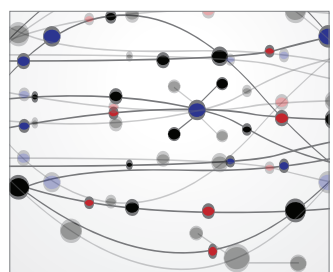

\section{The Scientific} World Journal
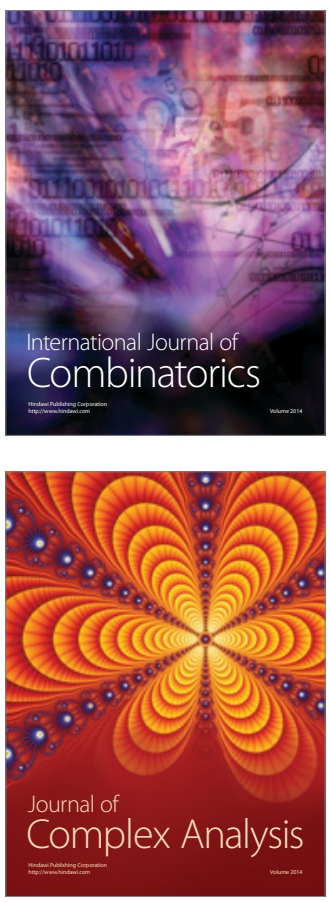

International Journal of

Mathematics and

Mathematical

Sciences
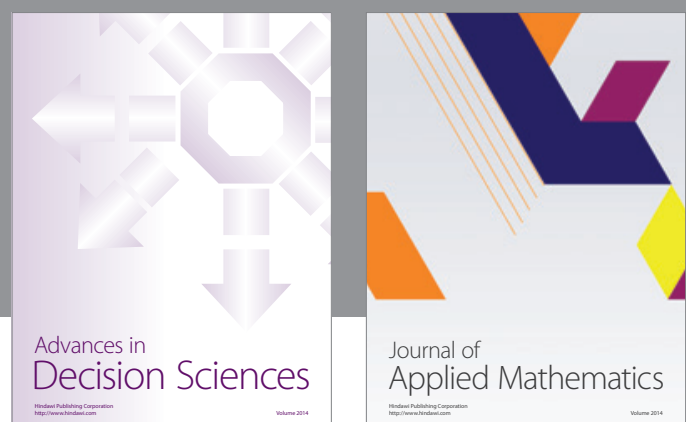

Journal of

Applied Mathematics
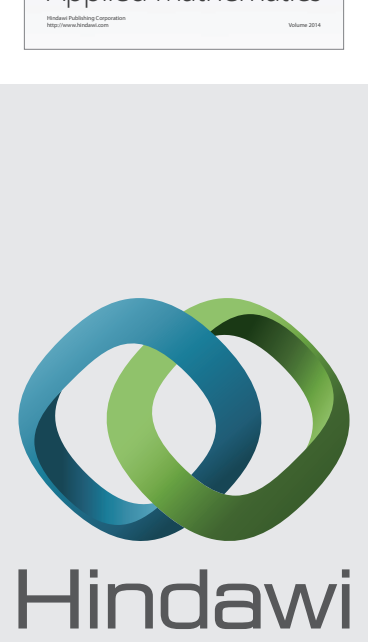

Submit your manuscripts at http://www.hindawi.com
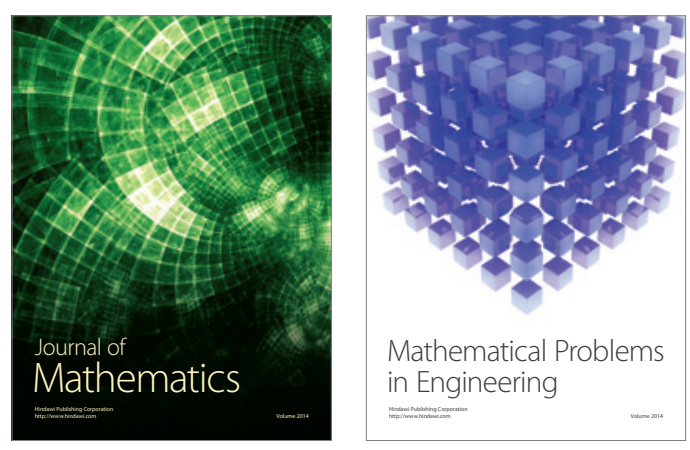

Mathematical Problems in Engineering
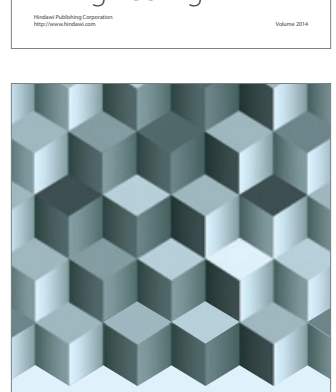

Journal of

Function Spaces
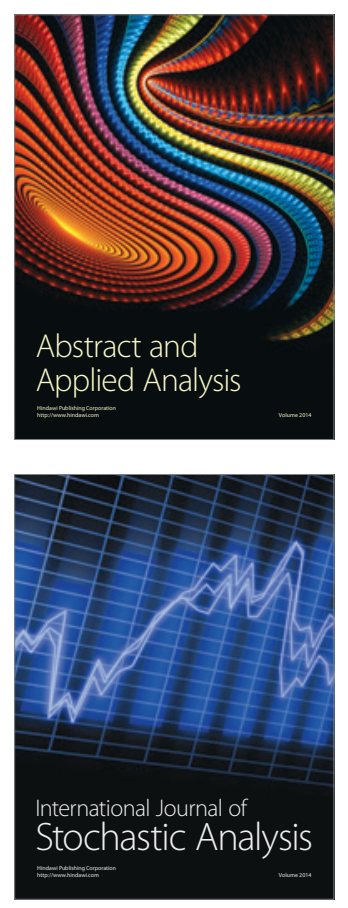

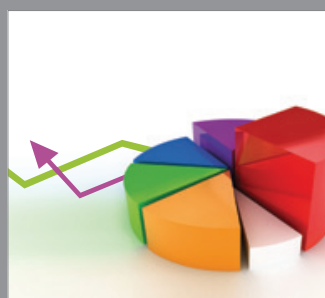

ournal of

Probability and Statistics

Promensencen
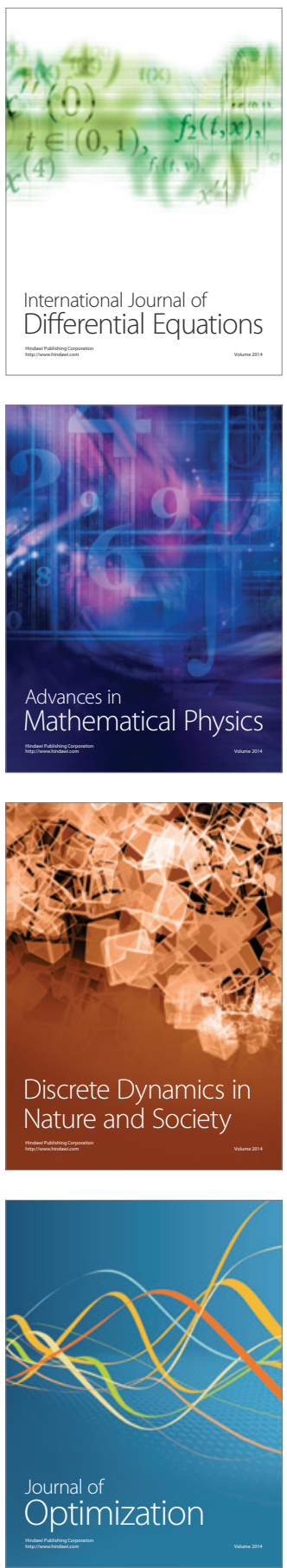